\title{
Educación STEM
}

en museos y escalas para valorarla

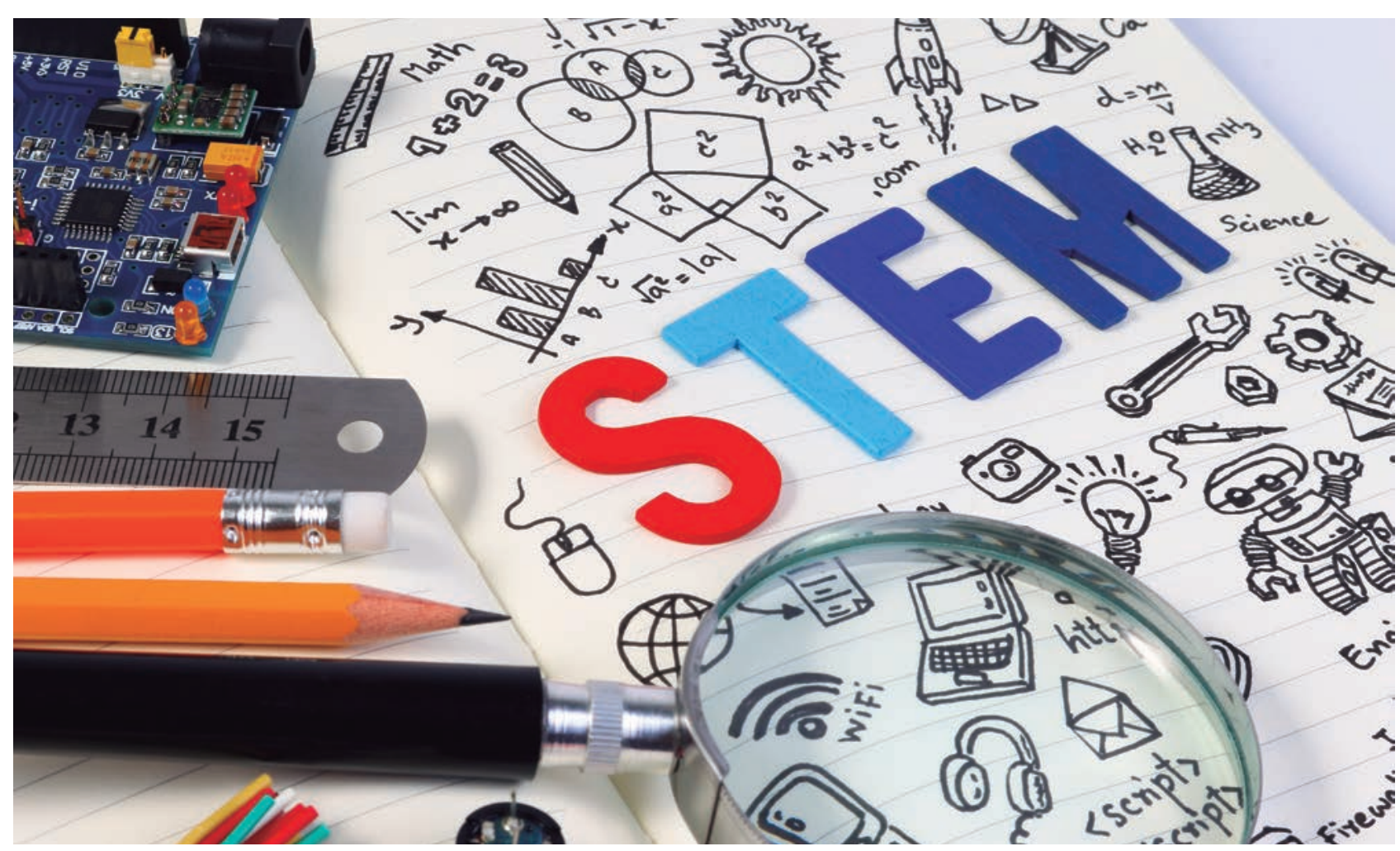

Dos maestras explican la experiencia que han tenido tras realizar su trabajo de fin de grado (TFG) durante el confinamiento y comparten su opinión acerca de la educación STEM como perspectiva del nuevo paradigma educativo. Para ello definen qué es, proponen escalas para su valoración e inciden en la importancia de su promoción en ámbitos educativos no

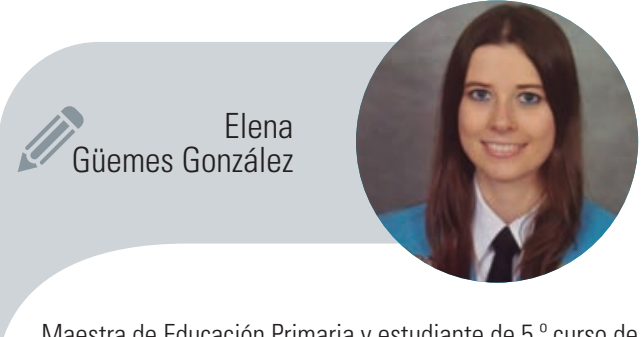

Maestra de Educación Primaria y estudiante de $5 .{ }^{\circ}$ curso de Educación Infantil elena.guemes@yahoo.es

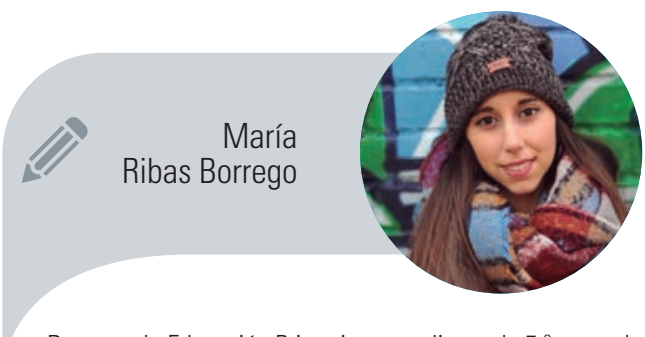

Docente de Educación Primaria y estudiante de $5 .^{\circ}$ curso de Educación Infantil maria.ribas@pepamayo.com formales. 
¿Quién nos iba a decir que tendríamos que realizar el proyecto que culmina nuestra carrera como maestras durante una pandemia mundial y ante un estado de alarma con el que todo el país debía permanecer en casa? Efectivamente, tuvimos que hacer nuestro trabajo de fin de grado (TFG) durante esos meses tan complicados en los que la incertidumbre estaba a la orden del día. También es de mucha actualidad el tema que escogimos nosotras: la educación STEM, acrónimo de las iniciales de las palabras en inglés Science, Technology, Engineering y Maths; Ciencias, Tecnología, Ingeniería y Matemáticas, respectivamente.

Tras varios meses de investigación y lecturas de artículos relacionados con este tema, somos conscientes de que vivimos en una sociedad globalizada que debe adaptarse a continuos cambios. Como consecuencia, es imprescindible un nuevo enfoque educativo en el que los docentes seamos capaces de preparar a los futuros ciudadanos para dar respuesta a las necesidades y las demandas que se presentarán en el futuro, teniendo en cuenta siempre que el objetivo final es conseguir el desarrollo personal y global de los alumnos en la sociedad. Por tanto, la educación debe apostar por un modelo interdisciplinario en el que se conecten y se apliquen todos los conocimientos necesarios para buscar la solución del reto presentado. Desde nuestro punto de vista, la educación STEM debe formar parte de dicho paradigma debido a que es una metadisciplina caracterizada por la integración de asignaturas que suelen ser trabajadas individualmente.

Diversos estudios demuestran que las profesiones relacionadas con el área STEM son las que tienen una mayor previsión de crecimiento. Por tanto, se requiere un aumento de la población especializada en estas áreas y dicha especialización resulta urgente, por lo que se debe comenzar a promover en los centros educativos programas que equipen a los alumnos con las aptitudes necesarias para las nuevas e, incluso, desconocidas profesiones de la sociedad

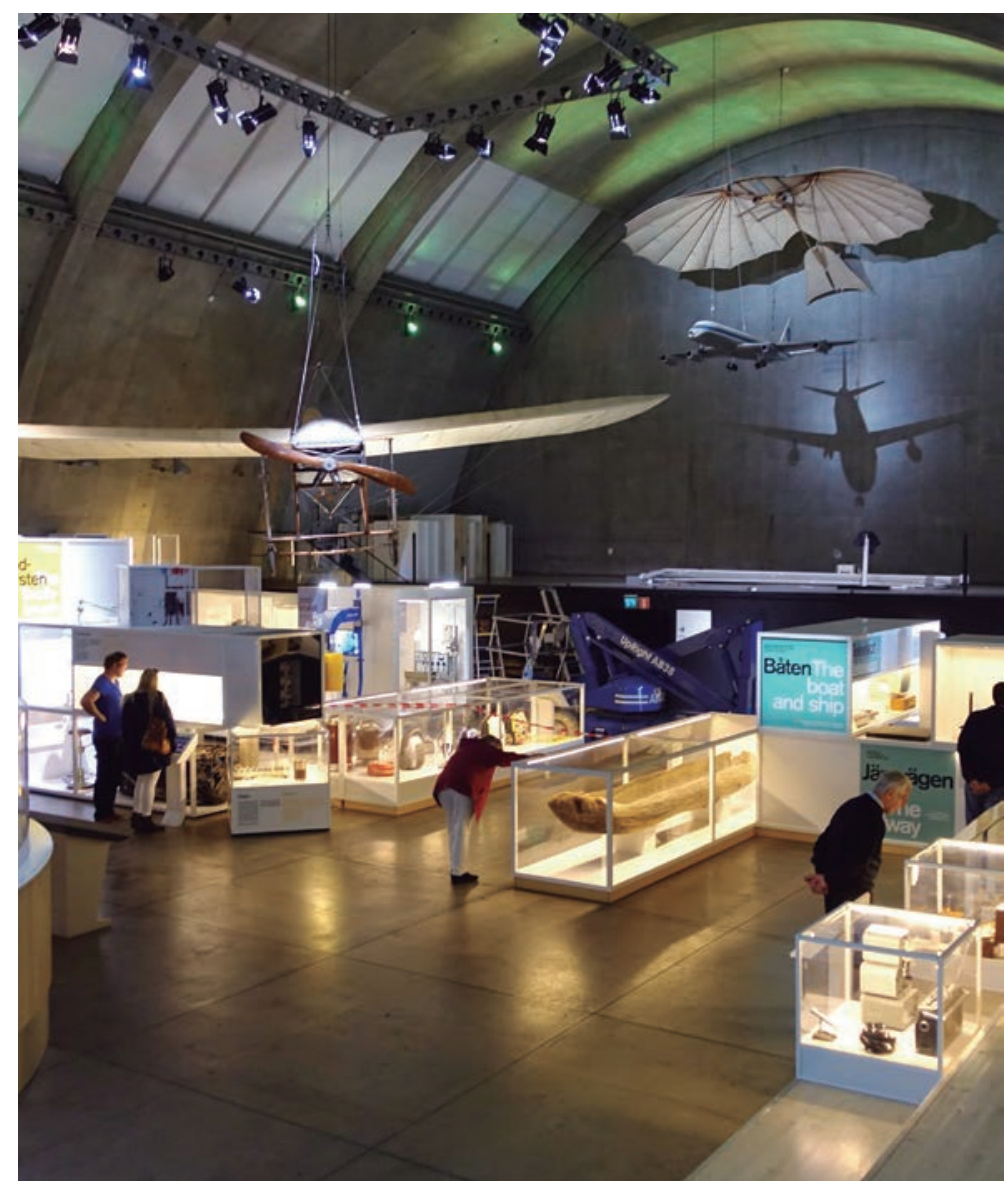

futura. No obstante, es una necesidad para todos, englobando a la totalidad de la nueva ciudadanía, pues no solo se va a requerir mayor mano de obra especializada, sino que permitirá participar en la toma de decisiones. Para ello, debemos comenzar a plantearnos cómo incluir la educación STEM en nuestras aulas y en otros ámbitos educativos no formales como, por ejemplo, parques o museos.

Teniendo en cuenta lo anterior, el ciudadano del siglo XXI debe adquirir $y$ desarrollar las competencias y habilidades específicas propias del mundo en el que vivimos actualmente. La educación STEM, a través del desarrollo de un aprendizaje competencial que dota al individuo de las habilidades imprescindibles para su desarrollo global a nivel profesional y general, trata de dar respuesta a este reto que se plantea.

Nosotras, como maestras, sabemos que tanto la etapa de Educación Infantil como la de Primaria son fundamentales para fomentar y desarrollar estas habilidades. Centrándonos más en las edades comprendidas de los 6 a los 12 años, en uno de los TFG se ha desarrollado un instrumento 
Habilidades para una sociedad eminentemente tecnológica

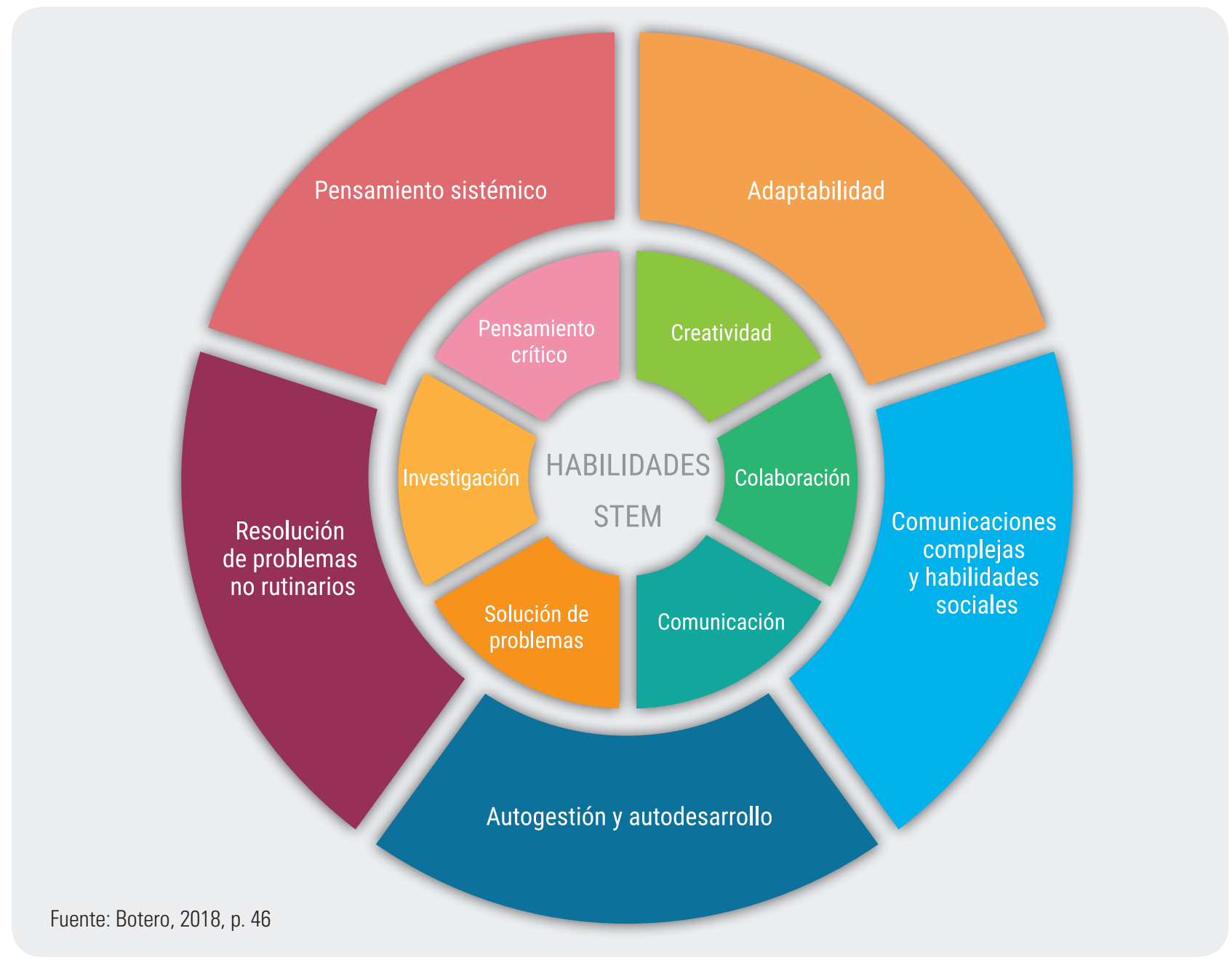

(una escala tipo Likert de cinco niveles) que permite no solo diseñar, sino también valorar las habilidades transversales que caracterizan una formación STEM. Así, estas últimas, forman las seis categorías de referencia: creatividad, colaboración, comunicación, resolución de problemas, pensamiento crítico e investigación. Además, cada una de ellas tiene asignados cuatro indicadores, lo que permite configurar un instrumento de valoración con 24 indicadores en total. Con el objetivo de representar de un modo visual y claro las puntuaciones asignadas a cada habilidad se propone un gráfico radial.

Posteriormente, en la segunda parte del TFG se realiza un análisis de una propuesta STEM ya diseñada, valorando así el grado de desarrollo de las distintas habilidades transversales en dicha práctica educativa de la etapa de primaria. De este modo, todo aquel que lea el TFG tiene un ejemplo práctico de cómo llevar a cabo la evaluación de las habilidades transversales propuestas STEM usando la escala diseñada para ello.

Todo lo presentado hasta ahora se enmarcaría dentro de la escuela. Sin embargo, la educación STEM puede Ilevarse a cabo en ámbitos no formales como pueden ser parques o museos. De este modo, el aprendizaje no debe detenerse al final de la jornada escolar, sino que debe continuar promoviéndose en otros ámbitos como pueden ser los de ocio y tiempo libre. Es en estos donde los niños y los jóvenes pueden desarrollar sus intereses y aficiones de manera prioritaria, ya que son momentos que se caracterizan por la ausencia de la presión escolar y la asimilación de los aprendizajes en la medida determinada por los estudiantes.

En relación con la educación no formal, se ha demostrado el impacto posi- 
tivo en la conflanza en disciplinas del área de la educación STEM que genera la participación en actividades extraescolares relacionadas con este ámbito. Como resultado, parques científicos y museos suponen una alternativa esencial para la aproximación a la ciencia de los jóvenes de cualquier edad.

En el otro TFG se realizó un estudio comparativo de la promoción de la educación STEM en ámbitos educativos no formales en España y en Suecia, dado que este último es uno de los países líderes en los resultados de las pruebas PISA. Los espacios comparados fueron el Tekniska Museet, que es el museo de ciencia de Estocolmo y el MUNCYT (Museo Nacional de Ciencia y Tecnología), en Alcobendas. Para ello, se seleccionaron cuatro dimensiones consideradas imprescindibles en los espacios makers, que corresponden a:

1. Grado de ludificación.

2. Grado de diversidad de los materiales, dispositivos y fenómenos con los que se trabaja.

3. Grado de promoción del trabajo colaborativo/cooperativo.

4. Grado de posibilidad de retroalimentación.

Además, se analizó la demanda cognitiva de la interacción a partir de varias categorías inspiradas en la taxonomía de Bloom más actuales.

Se compararon seis actividades similares propuestas por cada uno de los museos a través de los anteriores indicadores, así como los objetivos y contenidos que trabajaban, la retroalimentación que aportaba a los niños, la duración y el fundamento científico de la misma y las edades o cursos con los que se podría emplear.

Algunos ejemplos de las actividades escogidas en el Tekniska Museet para realizar la investigación fueron un vídeomicroscopio que permitía a los niños colocar algún objeto debajo de la lente para verlo ampliado en una pantalla. También había otro dispositivo que demostraba a los

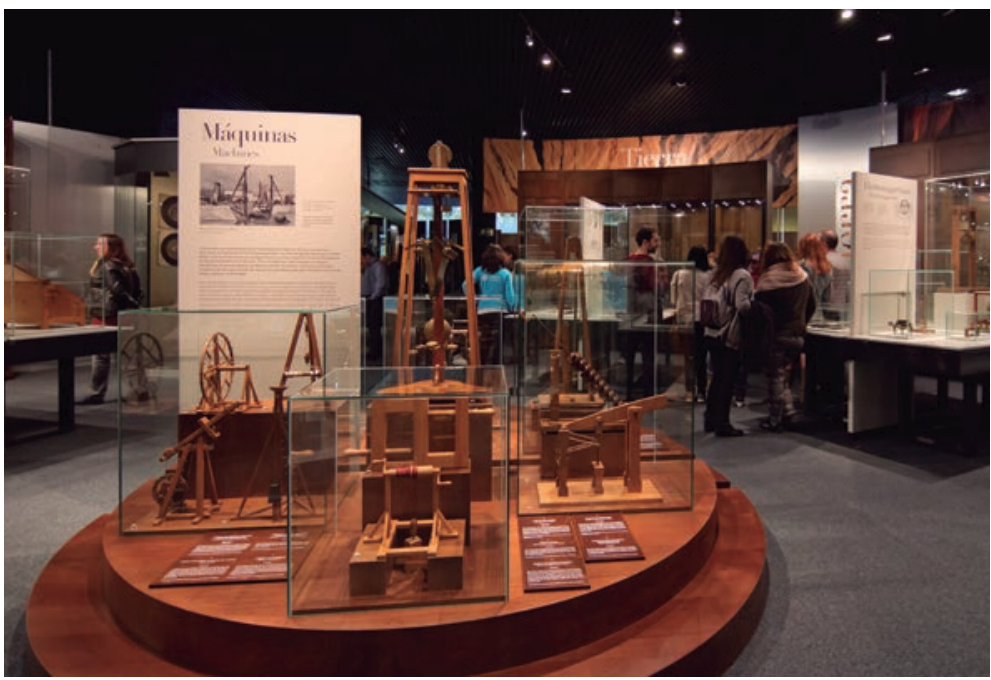

\section{Los niños y jóvenes que reciben desde edades rempranas una formación STEM rendrán una serie de competencias y habilidades específicas}

niños la teoría del Teorema de Pitágoras debido a que se encontraba un cuadrado grande lleno de líquido azul en la hipotenusa de un triángulo rectángulo y otros dos cuadrados más pequeños en los catetos. De esta manera, al girar el triángulo rectángulo se Ilenaban al completo los dos cuadrados pequeños con el líquido azul que se encontraba en el grande.

En el MUNCYT de Alcobendas, se seleccionaron algunos recursos que demostraban el Principio de Arquímedes y la funcionalidad de las poleas. En el primero se observaba un cilindro lleno de líquido en el que, al pulsar un botón, era introducido un peso dentro de dicho cuerpo geométrico, de manera que el líquido del interior aumentaba. Con respecto a las poleas, se proporcionaba una experiencia en la que los niños tienen que levantar varios sacos del mismo peso, pero la diferencia reside en la cantidad de poleas unidas a cada saco. Como resultado, cuando los niños intentan tirar de la cuerda que tiene una polea para levantar el saco, necesitan emplear más fuerza y esfuerzo que cuando tiran para levantar el que está conectado a dos o más poleas. 
En las siguientes tablas se puede comprobar el resultado de la investigación en la promoción de la educación STEM a partir de las actividades propuestas en ambos museos. A grandes rasgos los dos constan de un grado relativamente elevado de diversidad de materiales, dispositivos y fenómenos con los que experimentar. También, se caracterizan por su carácter lúdico y por la facilidad que presentan los dispositivos para que los jóvenes prueben y comprendan su fundamento científico.

Tabla 1. Valoración del Tekniska Museet como espacio que promueve la educación STEM

\begin{tabular}{l|l|l|l|l|l|l|} 
Actividad 1 & Actividad 2 & Actividad 3 & Actividad 4 & Actividad 5 & Actividad 6 & Grado final
\end{tabular}

1. Grado de diversidad de los materiales, dispositivos y fenómenos con los que trabajar

2. Grado de ludificación

3. Grado de posibilidad de retroalimentación

4. Grado de promoción del trabajo colaborativo/cooperativo

Medio

Fuente: elaboración propia

Tabla 2. Valoración del MUNCYT como espacio que promueve la educación STEM

\begin{tabular}{l|l|l|l|l|l|l} 
Actividad 1 & Actividad 2 & Actividad 3 & Actividad 4 & Actividad 5 & Actividad 6 & Grado final
\end{tabular}

1. Grado de diversidad de los materiales, dispositivos y fenómenos con los que trabajar

2. Grado de ludificación

3. Grado de posibilidad de retroalimentación

4. Grado de promoción del trabajo colaborativo/cooperativo

\begin{tabular}{|c|c|c|c|c|c|c|}
\hline Medio & Medio & Alto & Alto & Medio & Medio & Medio \\
\hline Bajo & Alto & Medio & Medio & Bajo & Medio & Medio \\
\hline Bajo & Medio & Alto & Alto & Bajo & Bajo & Medio \\
\hline Bajo & Bajo & Alto & Bajo & Bajo & Bajo & Bajo \\
\hline
\end{tabular}

Fuente: elaboración propia

En conclusión, los niños y jóvenes que reciben desde edades tempranas una formación STEM, tendrán una serie de competencias y habilidades específicas que sin duda serán necesarias para poder dar respuesta a los retos y situaciones que se les presenten en el futuro. Además, serán capaces de apli-

\section{(ㄷ) DARR SABER MẤS}

Armesto, F., Martínez losada, C., y García Barros, S. (2005). Museos como respuesta a las necesidades de formación de la ciudadanía. Revista Alambique, (43), 49-57.

Botero, J. (2018). STEM educación: introducción a una nueva forma de enseñar y aprender. Colombia: STEM Educación Colombia.

DomÈnECH-CASAL, J. (2018). Aprendizaje Basado en Proyectos en el marco STEM. Componentes didácticas para la Competencia Científica. Ápice. Revista de Educación Científica, 2(2), 29-42.

The Partnership For $21^{\text {st }}$ Century Learning (P21) (2017). $21^{\text {st }}$ Century Learning for Early Childhood Framework. Recuperado de http://static. battelleforkids.org/documents/p21/P21EarlyChildhoodFramework.pdf

car sus conocimientos a situaciones nuevas, así como de proseguir en la continua búsqueda de información. Todo este aprendizaje debe desarrollarse no solo en el ámbito académico formal, sino también en el no formal, porque tal y como afirmó la Organización de las Naciones Unidas para la Educación, la Ciencia y la Cultura (UNESCO, 1996, p. 6): "Los objetivos básicos de la educación deben ser aprender a aprender, aprender a resolver, aprender a ser" •

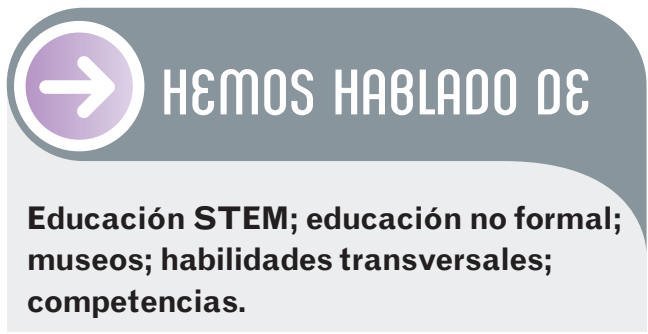

Este artículo fue solicitado por PADRES Y MAESTROS en mayo de 2020, revisado y aceptado en octubre de 2020. 